in either group in most of the physiological parameters examined; however, both total $K_{1}$ and CPCR-AA were significantly higher for those patients in both groups who survived sepsis. In vitro measurements confirmed that protein synthesis in tissue from survivors was significantly higher than in both normal liver tissue and tissue from those who died. No significant differences were found for any group in plasma PIF values; however, in survivors, there was a close correlation between PIF and CPCR-AA values. It is not clear whether lack of a correlation in those who recovered is due to the presence of a PIF inhibitor or whether there is a decrease in target tissue responsiveness. While the presence of increased amino acid is essential for survival, there is no apparent evidence that additional amino acid supplementation enhances the chance for survival.

K. Kasper, B.S.

\section{Estimation of True Calcium Absorption}

\author{
R. P. HEANEY, AND R. R. RECKER
}

Omaha, Nebraska

\section{Ann Intern Med 103: 516-521, 1985}

Measurements of true calcium absorption fraction in women were evaluated to see how closely calculations based on a single measurement of serum specific activity after administration of an oral calcium tracer might approximate the absorption value derived from the full, double-isotope absorption procedure. True absorption, body size, and miscible pool turnover could together explain better than $93 \%$ of the variance in serum calcium specific activity values 5 hours after a tracer-labeled test meal. Because measurement of pool turnover is not available routinely, it was dropped from the model, and a predictor equation was developed that allowed estimation of true absorption from the 5 -hour serum specific activity value, height, and weight. These variables explained $90.8 \%$ of the variance in the 5hour values and gave estimates of true absorption with a $95 \%$ confidence interval of \pm 0.055 . This small range of uncertainty makes the procedure useful in estimating absorption efficiency for calcium therapy in routine clinical practice.

Comment: The need for dietary calcium supplements especially in postmenopausal women is now well-recognized. Because of multiple homeostatic mechanisms aimed at maintaining calcium balance, serum concentrations of calcium do not reflect true absorption. Although many methods for the determination of calcium absorption have been described, most are of limited use clinically. It was shown that, in women, up to $93 \%$ of the variability in serum calcium specific activity at 5 and 7 hours after administration of a double-labeled calcium test meal could be accounted for by absorption fraction and to a lesser extent body surface area and turnover. Even with the elimination of turnover (which is not a readily available tool clinically) from the regression equation, $90.8 \%$ of the variance is still accounted for. This results in $95 \%$ confidence level of 0.055 of calcium per day, which although perhaps larger than desired, would be sufficient clinically to determine absorption of calcium and what range of supplement if any is indicated. Calcium absorption was studied only in women over age 35 ; therefore, caution should be exercised when applying the same regression to other age groups and men.

K. Kasper, B.S.

\section{Metabolism of Pantethine in Cystinosis}

\author{
C. T. WITTWER, W. A. GAHL, \\ J. DEB. BUTLER, M. ZATZ, AND \\ J. G. THOENE
}

Department of Pediatrics, University of Michigan Medical School, Ann Arbor, Michigan 48109; Interinstitute Genetics Program, Clinical Center, National Institutes of Health; Section on Biochemical Genetics, Human Genetics Branch, National Institute of Child Health and Human Development; and Laboratory of Cell Biology, National Institute of Mental Health, Bethesda, Maryland 20205

\section{J Clin Invest 76: 1665-1672, 1985}

D-Pantethine is a conjugate of the vitamin pantothenic acid and the low-molecular-weight aminothiol cysteamine. Pantethine is an experimental hypolipemic agent and has been suggested as a source of cysteamine in the treatment of nephropathic cystinosis. We treated four cystinotic children with 70$1,000 \mathrm{mg} / \mathrm{kg}$ per $\mathrm{d}$ oral D-pantethine and studied its metabolism. Pantethine was rapidly hydrolyzed to pantothenic acid and cysteamine; we could not detect pantethine in plasma after oral administration. The responsible enzyme, "pantetheinase," was highly active in homogenates of small intestinal mucosa and plasma. The Michaelis constant of the rat intestinal enzyme was $4.6 \mu \mathrm{M}$ and its $\mathrm{pH}$ profile showed a broad plateau between 4 and 9 . Pantothenate pharmacokinetics after orally administered pantethine followed an open two-compartment model with slow vitamin elimination $\left(t_{1 / 2}=28 \mathrm{~h}\right)$. Peak plasma pantothenate occurred at $2.5 \mathrm{~h}$ and levels over $250 \mu \mathrm{M}$ were seen at 300 times normal. Apparent total body storage of pantothenate was signficant $(25 \mathrm{mg} / \mathrm{kg})$, and plasma levels were elevated three- 
fold for months after pantethine therapy. Plasma cysteamine concentrations after pantethine were similar to those reported after equivalent doses of cysteamine. However, at best only $80 \%$ white blood cell cystine depletion occurred. We conclude that pantethine is probably less effective than cysteamine in the treatment of nephropathic cystinosis and should only be considered in cases of cysteamine intolerance. Serum cholesterol was decreased an average of $14 \%$, which supports the potential clinical significance of pantethine as a hypoplipemic agent. Rapid in vivo hydrolysis of pantethine suggests that pantethenate or cysteamine may be the effectors of its hypolipemic action.

Comment: Pantethine, a conjugate of panthothenic acid and cysteamine, was examined as a source of cysteamine for the treatment of nephrotic cystinosis. In this disease defective lysosomal membrane transport systems result in an accumulation of cellular cystine. The treatment of choice for this disease has been administration of oral doses of cysteamine which converts cystine into products which can freely leave the cell. However, panthethine is more palatable and less toxic to the system than cysteine. Upon administration of panthetine, plasma cysteamine increased in a dose-related manner. Pantethine plasma levels were undetectable indicating that pantethine was almost entirely hydrolyzed at the intestinal wall rather than at the cellular level. This indicates that pantethine is not more efficient in cystinosis than cysteine. In fact the converse appears to be true. Cysteamine can deplete $\mathbf{> 9 0} \%$ of leukocyte cystine whereas pantethine resulted in a maximum $80 \%$ depletion. Pantethine is therefore only indicated in patients who cannot tolerate cysteine therapy.

K. Kasper, B.S. 